\title{
Increased Plasma Levels of Soluble Intercellular Adhesion Molecule-1 (sICAM-1) and Soluble Vascular Cell Molecule-1 (sVCAM-1) Associated with Disease Severity in a Primate Model for Severe Human Malaria: Plasmodium coatneyi-Infected Japanese Macaques (Macaca fuscata)
}

\author{
Satoru KAWAI ${ }^{1)}$, Jun MATSUMOTO ${ }^{1)}$, Masamichi AIKAWA ${ }^{2)}$ and Hajime MATSUDA ${ }^{1)}$ \\ ${ }^{1)}$ Department of Tropical Medicine and Parasitology, Dokkyo University School of Medicine, Mibu, Tochigi 321-0293 and ${ }^{2)}$ Graduate \\ School of Agricultural and Life Sciences, The University of Tokyo, 1-1-1 Yayoi, Bunkyo-ku, Tokyo 113-8657, Japan
}

(Received 25 September 2002/Accepted 17 January 2003)

ABSTRACT. In the present study, we investigated plasma levels of soluble intercellular adhesion molecule-1 (sICAM-1) and vascular cell adhesion molecule-1 (sVCAM-1) in seven Japanese macaques (Macaca fuscata) infected with Plasmodium coatneyi. Concentrations of sICAM-1 and SVCAM-1 were significantly elevated in the severe phase; the levels were maximally increased up to six times and three times those before infection, respectively. We subsequently examined kinetic profiles of sICAM- 1 and sVCAM- 1 concentration in plasma obtained from two infected monkeys. Both infected monkeys had markedly increased levels of these adhesion molecules when they exhibited severe clinical signs correlated with rapid increase in parasitemia. These results suggest that the elevation of levels of sICAM-1 and sVCAM-1 is a critical step in the pathogenesis of severe malaria in vivo.

KEY wORDs: Plasmodium coatneyi, sICAM-1, sVCAM-1.

J. Vet. Med. Sci. 65(5): 629-631, 2003

The sequestration of infected red blood cells (IRBCs) in various organs is a major factor in Plasmodium falciparum infection leading to severe malaria [12]. The cytoadherence of IRBCs causing sequestration results from specific interactions between ligands on the surface of IRBC and counter-receptors expressed on endothelial cells [6, 15]. The participation of intercellular adhesion molecule-1 (ICAM-1) and vascular cell adhesion molecule-1 (VCAM1), which are principal candidates for this interaction, have been elucidated in vitro, and expression of them has been identified in postmortem tissues from fatal falciparum malaria [6]. It is therefore that these adhesion molecules play important roles in the pathogenesis of vital organ dysfunction during the multiple complications associated with $P$. falciparum infection.

Expression of ICAM-1 and VCAM-1 on endothelial cells can be upregulated by proinflammatory cytokines, such as tumour necrosis factor (TNF) and interleukin 1 (IL-1 $\alpha$ ), and is accompanied by increased release of a soluble form of the adhesion molecule [7]. In previous studies of Gambian children and Thai patients with $P$. falciparum malaria, plasma levels of soluble (s) ICAM-1 and sVCAM-1 were significantly increased and correlated positively with the severity of malaria [8, 13]. Boehme et al. have also described an increase in plasma concentrations of sICAM-1, sVCAM-1 and $\mathrm{sE}$-selectin in patients suffering from severe malaria, compared with the concentrations in a group of healthy volunteers [4]. However, not all studies have confirmed a relationship between severity of malaria and levels of soluble adhesion molecules, and the kinetics of these molecules over the course of infection remains unclear [14].

$P$. coatneyi is a simian malaria parasite of macaque monkeys with a number of falciparum-like characteristics. Pre- vious studies have shown that the clinical and pathologic characteristics of experimental hosts infected with $P$. coatneyi, such as rhesus monkeys (Macaca mulatta) and Japanese macaques (M. fuscata), appear similar to those of severe falciparum malaria in human $[1,9]$. Therefore, $P$. coatneyi-infected experimental hosts are thought to offer a useful model for pathological and pathophysiological studies of severe falciparum malaria. The purpose of this study was to investigate how the changes in plasma levels of sICAM-1 and SVCAM-1 in this primate model were linked to the course of infection by malaria.

Seven Japanese macaques were used in this study (Table 1). All monkeys were bred and grown in animal facilities in Japan. The investigators adhere to the Guidelines for the Use of Experimental Animals authorized by the Japanese Association for Laboratory Animal Science. The infected monkeys were inoculated intravenously with about $1 \times 10^{9}$ frozen $P$. coatneyi-infected erythrocytes (CDC strain). After inoculation, daily clinical follow-up of the monkeys

\begin{tabular}{ccccc} 
Table 1. & \multicolumn{5}{l}{ Summary of experimental animals } \\
\hline $\begin{array}{c}\text { Animal } \\
\text { No. }\end{array}$ & $\begin{array}{c}\text { Age } \\
\text { (year) }\end{array}$ & Sex & $\begin{array}{c}\text { Autopsy } \\
\text { performed on day }\end{array}$ & $\begin{array}{c}\text { Parasitemia (\%) } \\
\text { at autopsy }\end{array}$ \\
\hline J-6 & 1 & F & 13 & 14.0 \\
J-7 & 2 & F & 11 & 15.6 \\
J-8 & 2 & M & 14 & 30.6 \\
J-9 & 2 & M & 12 & 41.0 \\
J-10 & 1 & F & 12 & 29.7 \\
J-11 & 1 & M & 11 & 17.6 \\
J-12 & adult & F & 19 & 13.1 \\
\hline mean \pm SD & & $13.1 \pm 2.6$ & $32.1 \pm 9.9$ \\
\hline
\end{tabular}

adult: $>10$ years $\mathrm{F}$ : female $\mathrm{M}$ : male 
was performed over the course of infection. From the 6th day after infection, thin blood films were prepared daily from peripheral blood obtained by earprick and Giemsastained, and development of parasites in the infected monkeys was monitored by microscopic observation.

Blood samples from the infected monkeys were obtained on day 0 and every 3 or 4 days after infection. All animals were anesthetized by an intramuscular injection of ketamine hydrochloride (10-20 mg/kg) when they became lethargic and severely withdrawn, and were then exsanguinated for autopsy.

Plasma levels of sICAM-1 and sVCAM-1 were determined using a commercial enzymatic immunoassay kit (R \& D Systems Europe, Abingdon, UK). Seven infected monkeys were used for assays on day 0 and at severe phase. In addition, the kinetics of plasma SICAM-1 and sVCAM-1 concentration were investigated using two (J6 and J7) of the seven infected monkeys. Estimation of these adhesion molecules in severe phase was performed for plasma samples just before autopsy. The significance of differences between day 0 and severe phase in the infected Japanese macaques was tested by Student's paired- $t$ test. $P$-values lower than 0.05 were considered significant.

The parasite was first detected in the peripheral blood of the infected Japanese macaques about 8 days after infection; parasite densities then increased sharply by a day or two prior to autopsy. Mean parasitemia at autopsy was $32.1 \pm$ 9.9\% (Table 1). The infected monkeys initially tolerated malarial infection without any behavioral changes. Subsequent clinical signs ranged from partial anorexia to severe manifestations, such as complete anorexia, restlessness and depression, correlated with the rapid increase in parasitemia. The infected Japanese macaques other than J12 finally became lethargic and comatose within 14 days after infection, and J12 eventually became moribund on the 19th day after infection (Table 1).

Figure 1 summarizes the plasma levels of sICAM-1and sVCAM-1 in the seven infected Japanese macaques. Significant increase in plasma levels of sICAM-1and SVCAM-1 was found in all infected Japanese macaques in severe phase. On day 0 , the mean plasma levels of sICAM- 1 and sVCAM- 1 were $566.6 \pm 152.5 \mathrm{pg} / \mathrm{m} l$ and $181.8 \pm 91.9 \mathrm{ng} / \mathrm{m} l$ (mean $\pm \mathrm{SD}$ ), respectively. The concentrations of sICAM-1 significantly increased in severe phase compared to plasma levels on day $0(2372.9 \pm 1067.8 \mathrm{pg} / \mathrm{ml}, p<0.005)$, and exhibited maximal increase of up to 6 times (animal No. J12). The concentrations of SVCAM-1 were also significantly increased in severe phase $(470.5 \pm 295.6 \mathrm{ng} / \mathrm{ml}$, $p<0.05$ ), and exhibited a maximal increase of up to 3 times (animal No. J7).

We subsequently measured the kinetics of plasma sICAM-1 and sVCAM-1 concentrations over the course of infection obtained for two infected monkeys (J6 and J7). As clearly shown in Fig. 2, both infected monkeys had markedly increased levels of sICAM-1 and sVCAM-1 during later infection, when they exhibited severe clinical signs correlated with the rapid increase in parasitemia.
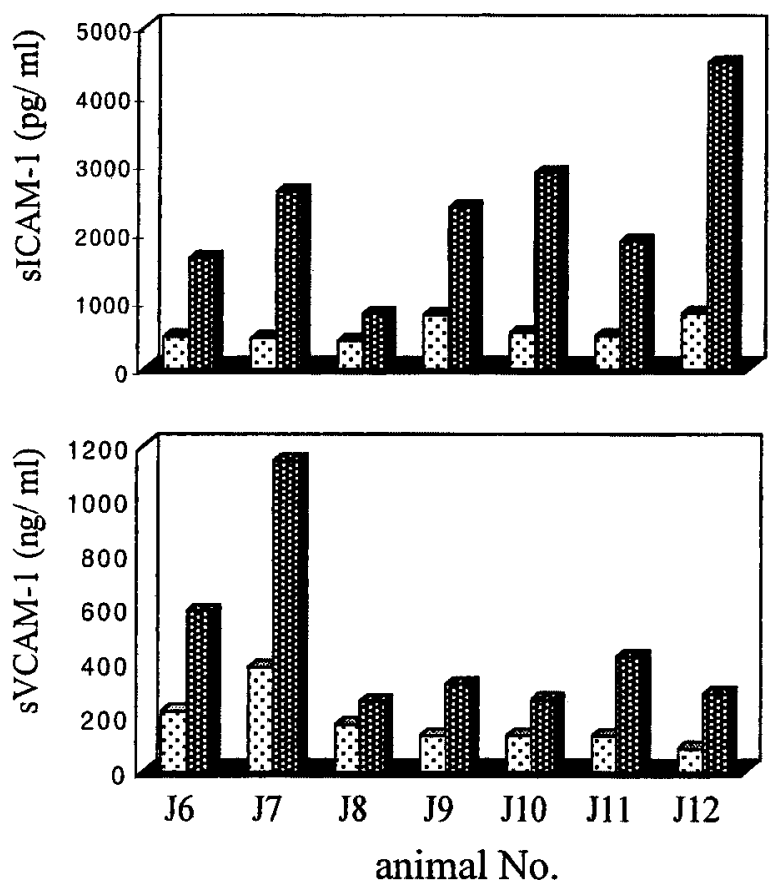

Fig. 1. Changes in plasma levels of soluble adhesion molecules in Plasmodium coatneyi-infected Japanese macaques between day 0 ( sICAM-1 and sVCAM-1 were significantly elevated in severe phase; the levels were maximally increased up to six times (animal No.J12) and three times (animal No.J7) those before infection, respectively. $P$-values were determined by Student's paired- $t$ test.

Higher expression of adhesion molecules and production of proinflammatory cytokines have been found in humans with cerebral and severe malaria than in those with uncomplicated malaria [4, 7, 8, 10, 13, 14]. Several investigators have pointed out that high levels of TNF and IL-1- $\alpha$ production may exacerbate IRBC sequestration by upregulating ICAM-1 and VCAM-1 expression on endothelium, and that this may be a critical step in the pathogenesis of severe human malaria with cerebral involvement [3]. However, it has proven difficult to test this hypothesis in vivo.

In this study, we found a marked elevation of soluble adhesion molecules in $P$. coatneyi-infected Japanese macaques in the severe phase. Moreover, the kinetics of sICAM-1 and sVCAM-1 were positively correlated with parasitemia and the occurrence of severe clinical signs. In a previous study using $P$. coatneyi-infected rhesus monkeys, increase in plasma levels of TNF- $\alpha$, IL- $1 \beta$ and IFN- $\gamma$ was related to increased parasitemia over the course of later infection [17]. We have also observed increase in plasma levels of TNF- $\alpha$ and IFN- $\gamma$ in P. coatneyi-infected Japanese macaques in the severe phase (unpublished data). The increased production of these cytokines probably resulted from monocyte/macrophage activation since monocytes/ macrophages activated by stimulation with parasitic antigens are a major source of both TNF and IL-1 [2, 5]. In vitro 


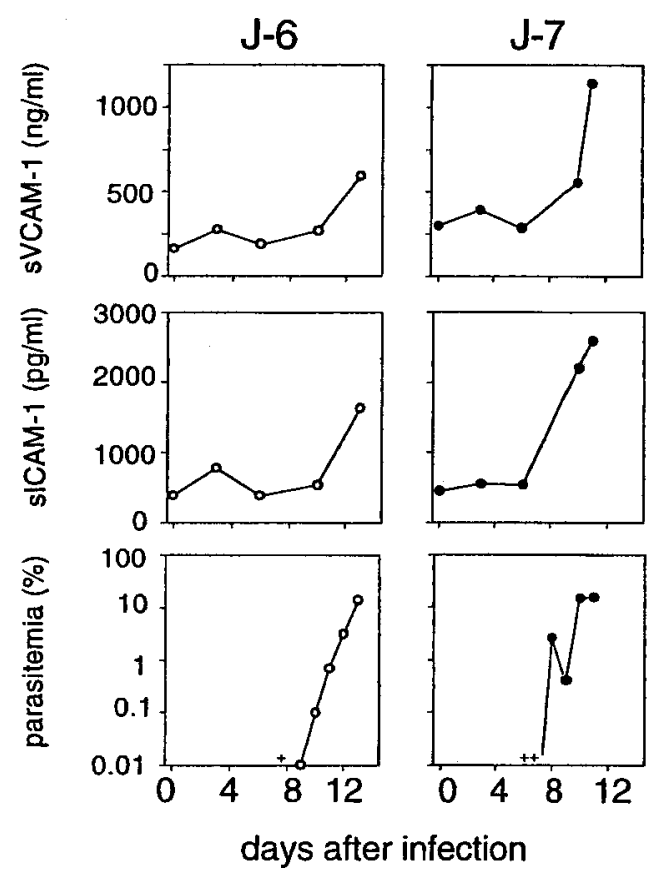

Fig. 2. Kinetics of parasitemia, plasma sICAM-1 and sVCAM-1 levels in two infected Japanese macaques (J6 and J7) over the course of infection. Both infected monkeys had markedly increased levels of these adhesion molecules correlated with disease severity.

studies on the cellular source of sICAM-1 and sVCAM-1 have shown that the release of soluble form correlates with levels of ICAM-1 and VCAM-1 expressed on endothelium in response to TNF and IL-1 $[11,16]$. Therefore, the markedly elevated levels of soluble adhesion molecules in $P$. coatneyi-infected Japanese macaques in the severe phase might reflect enhanced interaction between IRBCs and cytokine activated endothelial cells with subsequent shedding. The elevation in levels of SICAM-1 and SVCAM-1 with disease severity are compatible with the view that expression of high levels of these adhesion molecules is a critical step in the pathogenesis of severe malaria in vivo.

We are very grateful to Dr. William E. Collins (Centers for Disease Control and Prevention) for kindly providing the
P. coatneyi strain. This study was supported by a Grant-inAid for Scientific Research on Priority Areas (No.08281103, No. 12670239) from the Ministry of Education, Science, Culture and Sports, Japan.

\section{REFERENCES}

1. Aikawa, M., Brown, A., Smith, C.D., Tegoshi, T., Howard, R.J., Hasler, T.H., Ito, Y., Perry, G., Collins, W.E. and Webster, K. 1992. Am. J. Trop. Med. Hyg. 46: 391-397.

2. Bate, C.A.W., Traverne, J. and Playfair, J.H.L. 1988. Immunology 64: 227-231.

3. Berendt, A.R., Ferguson, D.J.P., Tansey, J., Newbold, C.I. and Marsh, K. 1990. Parasitol. Today 6: 247-254.

4. Boehme, M.W.J., Werle, E., Kommerell, B. and Raeth, U. 1994. Clin. Invest. 72: 598-603.

5. Clark, I.A., Rockett, K.A. and Cowden, W.B. 1991. Parasitol. Today 7: 205-207.

6. Fujioka, H. and Aikawa, M. 1996. Micro. Pathog. 20: 63-72.

7. Gearing, A.J.H. and Newman, W. 1993. Immunol. Today 14: 506-512.

8. Jakobsen, P.H., Morris-Joness, S., Rønn, A., Hviid, L., Theander, T.G., Elhassan, I.M., Bygbjerg, I.C. and Greenwood, B.M. 1994. Immunology 83: 665-669.

9. Kawai, S., Aikawa, M., Kano, S. and Suzuki, M. 1993. Am. J. Trop. Med. Hyg. 48: 630-636.

10. Kwiatkowski, D., Hill, A.V.S., Sambou, I., Twumasi, P., Castracane, J., Manogue, K.R., Cerami, A., Brewster, D.R. and Greenwood, B.M. 1990. Lancet 336: 1201-1204.

11. Leeuwenberg, J. F. M., Smeets, E. F., Neefjes, J. J., Shaffer, M.A., Clinek, T., Jeunhomme, T. M. A. A., Ahern, T. J. and Buurman, W. A. 1992. Immunology 77: 543-549.

12. Miller, L.H., Good, M.F. and Milon, G. 1994. Science 264: 1878-1883.

13. Muanza, K., Traoré, B., Gay, F., Krudsood, S., Danis, M. and Looareesuwan, S. 1999. Ann. Trop. Med. Parasitol. 93: 449455.

14. McGuire, W., Hill, A.V.S., Greenwood, B.M. and Kwiatkowski, D. 1996. Tras. Roy. Soc. Trop. Med. Hyg. 90: 274276.

15. Ockenhouse, C.F., Tegoshi, T., Maeno, Y., Benjamin, C., Ho, M., Kan, K.E., Thway, Y., Win, K., Aikawa, M. and Lobb, R.R. 1992. J. Exp. Med. 176: 1183-1189.

16. Pigott, R., Dillon, L.P., Hemingway, I.K. and Gearing, A.J.H. 1992. Biochem. Biophys. Res. Commun. 187: 584-589.

17. Yang, C., Xiao, L., Tongren, J.E., Sullivan, J., Lal, A.A. and Collins, W.E. 1999. Am. J. Trop. Med. Hyg. 61: 226-229. 